\title{
Stellar population synthesis of post-AGB stars: the s-process in MACHO 47.2496.8
}

\author{
A. Bonačić Marinović ${ }^{1}$, M. Lugaro ${ }^{1,2}$, M. Reyniers ${ }^{3, \star}$, and H. Van Winckel ${ }^{3}$ \\ 1 Sterrekundig Instituut, Universiteit Utrecht, PO Box 80000, 3508 TA Utrecht, The Netherlands \\ e-mail: [A.A.BonacicMarinovic; M.Lugaro]@astro.uu.nl \\ 2 Centre for Stellar and Planetary Astrophysics, School of Mathematical Sciences, Monash University, Victoria 3800, Australia \\ 3 Instituut voor Sterrenkunde, Departement Natuurkunde en Sterrenkunde, K. U. Leuven, Celestijnenlaan 200D, 3001 Leuven, \\ Belgium \\ e-mail: [maarten.reyniers; hans.vanwinckel]@ster.kuleuven.be
}

Received 11 June 2007 / Accepted 6 July 2007

\section{ABSTRACT}

\begin{abstract}
Context. The low-metallicity RV Tauri star MACHO 47.2496.8, recently discovered in the Large Magellanic Cloud, is highly enriched in carbon and heavy elements produced by the slow neutron capture process ( $s$-process), and is most probably a genuine post-C(N-type) asymptotic giant branch (AGB) star. The intrinsic interpretation of the enrichement is further strengthened by detection of a significant infrared excess. The circumstellar dust is the relic of a recent episode of heavy mass loss. We use the analysis of the abundances of MACHO 47.2496.8 to constrain free parameters in AGB models.

Aims. We test which values of the free parameters describing uncertain physical mechanisms in AGB stars, namely the third dredgeup and the features of the ${ }^{13} \mathrm{C}$ neutron source, produce models that better match the abundances observed in MACHO 47.2496.8. Methods. We carry out stellar population synthesis coupled with $s$-process nucleosynthesis using a synthetic stellar evolution code. Results. The $s$-process ratios observed in MACHO 47.2496.8 can be matched by the same models that explain the $s$-process ratios of Galactic AGB and post-AGB stars of metallicity $>Z_{\odot} / 10$, except for the choice of the effectiveness of ${ }^{13} \mathrm{C}$ as a neutron source, which has to be lower by roughly a factor of 3 to 6 . The less effective neutron source for lower metallicities is also required when comparing population synthesis results to observations of Galactic halo $s$-enhanced stars, such as $\mathrm{Pb}$ stars. The ${ }^{12} \mathrm{C} /{ }^{13} \mathrm{C}$ ratio in MACHO 47.2496.8 cannot be matched simultaneously and requires the occurrence of extra-mixing processes.

Conclusions. The confirmed trend of the decreased efficiency of the ${ }^{13} \mathrm{C}$ neutron source with metallicity requires an explanation from AGB $s$-process models. The present work is to date the first comparison between theoretical models and the detailed abundances of an extragalactic post-AGB star.
\end{abstract}

Key words. nuclear reactions, nucleosynthesis, abundances - stars: AGB and post-AGB - stars: abundances galaxies: Magellanic Clouds - stars: individual: MACHO 47.2496.8

\section{Introduction}

Slow neutron capture ( $s$-process) elements are synthesized in the intershell region between the $\mathrm{C}-\mathrm{O}$ core and the convective $\mathrm{H}$-rich envelope of asymptotic giant branch (AGB) stars. The main neutron source is believed to be ${ }^{13} \mathrm{C}$ nuclei, releasing neutrons via the ${ }^{13} \mathrm{C}(\alpha, n){ }^{16} \mathrm{O}$ reaction in a thin layer of the intershell (the ${ }^{13} \mathrm{C}$ pocket, see e.g. Gallino et al. 1998, for details). The $s$-process elements are mixed to the stellar surface by recurrent episodes of third dredge-up (TDU), where they are observed. A high abundance of ${ }^{13} \mathrm{C}$ in the pocket can be produced by ${ }^{12} \mathrm{C}+p$ reactions if protons are mixed from the convective envelope into the radiative ${ }^{12} \mathrm{C}$-rich intershell. This mixing would likely occur at the end of each TDU episode, when a sharp discontinuity is left between the convective and the radiative regions. However, it is not yet clear what mechanism produces this mixing. The extent of the mixing, which makes the effectiveness of the ${ }^{13} \mathrm{C}$ neutron source, is the most uncertain parameter in $s$-process models (see e.g. Busso et al. 1999; Lugaro et al. 2003; Herwig 2005, for discussion).

\footnotetext{
^ Postdoctoral fellow of the Fund for Scientific Research, Flanders.
}

Post-AGB stars are in the fast evolutionary phase between the AGB and white dwarf tracks. The strong mass loss at the end of the AGB has stopped, but they are not yet hot enough to ionise their circumstellar medium and to produce a planetary nebula. Their elemental abundances are the result of their evolutionary history, thus they can be used to probe and constrain the nucleosynthesis that takes place in AGB stars, in particular the $s$-process.

A peculiar post-AGB object, MACHO 47.2496.8, was found in the Large Magellanic Cloud (LMC) by Pollard \& Lloyd Evans (2000) and analysed in detail by Reyniers et al. (2007). Its main features are a very low metallicity $([\mathrm{Fe} / \mathrm{H}]=-1.4)$, excess of carbon over oxygen $\left(\mathrm{C} / \mathrm{O}>2\right.$ with $\left.{ }^{12} \mathrm{C} /{ }^{13} \mathrm{C}=200 \pm 25\right)$, an enhancement of 1.2 dex of light $s$-process elements (ls) compared to iron, a strong enhancement of heavy $s$-process elements (hs) compared to iron of $2.1 \mathrm{dex}$, and a similar enhancement of lead over iron. In Reyniers et al. (2007), it was argued that the luminosity $\left(\sim 5000 L_{\odot}\right)$ and the specific pulsational behaviour (RV Tauri like) of MACHO 47.2496.8 favour an intrinsic origin of the $s$-process enrichement, although an extrinsic scenario in which the enrichment is caused by a former AGB companion, now on the white dwarf track, could not be excluded. 


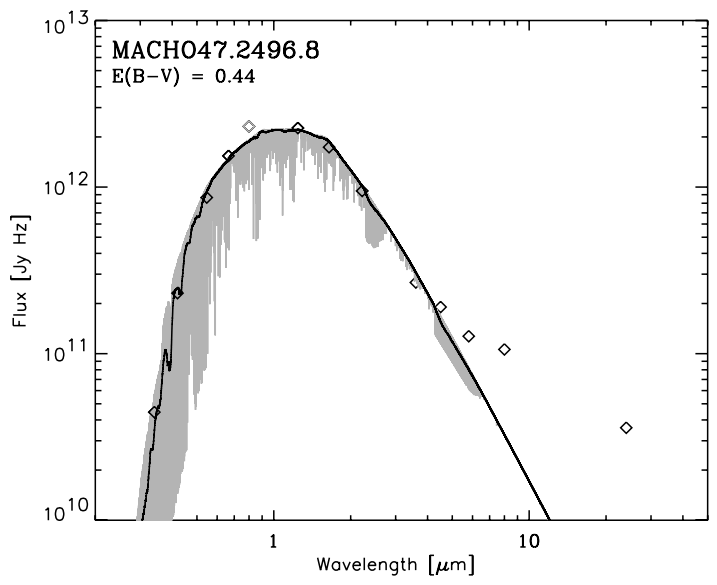

Fig. 1. The spectral energy distribution of MACHO 47.2496.8 as in Reyniers et al. (2007), but updated with Spitzer SAGE fluxes, revealing the presence of circumstellar dust. Diamonds are the measured magnitudes (from blue to red): Geneva $U, B, V$ and Cousins $R$ taken with C2+Euler; $I$ from DENIS (in gray), SAAO $J, H, K$, and the Spitzer SAGE fluxes $(3.6,4.5,5.8,8.0$, and $24 \mu \mathrm{m})$. The MARCS model is shown in gray, while a smoothed version is shown with a full black line. The $I$ magnitude could not be fitted, possibly due to a phase difference.

We have updated the Spectral Energy Distribution (SED) of MACHO 47.2496.8 presented in Reyniers et al. (2007) by adding the newly released Spitzer SAGE data (Meixner et al. 2006) of the source. The new data reveal a small but clear infrared excess starting around $5 \mu \mathrm{m}$ (Fig. 1). In the Galaxy, the RV Tauri pulsators with a dust excess are dominated by binaries, consisting of a post-AGB primary and an unevolved companion. In these systems, the dust is trapped in a stable circumbinary disc (De Ruyter et al. 2006). The LMC RV Tauri stars detected by the Macho experiment are also dominated by disc sources (Reyniers \& Van Winckel 2007) and the SAGE data reveal that the typical colours yield a $K-[5.8 \mu \mathrm{m}]>2.5$ and $[8 \mu \mathrm{m}]-[24 \mu \mathrm{m}]$ indicative of a dust excess with a hot dust component.

The colours of MACHO 47.2496.8 $(K-[5.8]=0.8$ and $[8]-[24]=2.4)$ are significantly different, showing that the excess is much colder than in the suspected disc sources. We conclude that the dust excess of MACHO 47.2496.8 corroborates the post-AGB status of the object in which the excess is a relic of a recent phase of dusty mass loss. The object is an ideal source to study the dust formation in metal poor conditions. In principle, it could still represent the post-AGB phase of a former, extrinsically enriched, $\mathrm{CH}$ star (about $5 \%$ of the carbon stars in the Stephenson 1989, catalog are extrinsically s-process and carbon enriched $\mathrm{CH}$-stars) but we consider that it is much more likely that the object is a genuine intrinsically enriched post-AGB star.

In this contribution, we focus on the modelling of the chemical content assuming that the object is indeed intrinsically enriched. By comparing recent stellar population calculations including nucleosynthesis of $s$-process elements to observations of Galactic stars, Bonačić Marinović et al. (2007, hereafter Paper I) have set constraints on several free parameters included in their models: the minimum core mass for TDU, the TDU efficiency $(\lambda)$, the effectiveness of the ${ }^{13} \mathrm{C}$ neutron source $\left({ }^{13} \mathrm{C}_{\mathrm{eff}}\right)$, and the size in mass of the ${ }^{13} \mathrm{C}$ pocket. In this paper we use those models to interpret the abundances of MACHO 47.2496.8 and determine whether they can be reproduced using the same set of values of the free parameters found in Paper I.

\section{Stellar population synthesis models}

We calculated populations of post-AGB stars with our rapid synthetic stellar evolution code, which includes $s$-process nucleosynthesis based on the models of Gallino et al. (1998). This code is described in detail in Paper I and the population synthesis procedure is done in the same fashion as in Paper I.

We run our models on a grid of 50 metallicity values, $[\mathrm{Fe} / \mathrm{H}]$, linearly separated, in the range $-2.0<[\mathrm{Fe} / \mathrm{H}]<-0.5$ and 500 initial masses, $M_{\mathrm{i}}$, logarithmically separated, in the range $0.7 M_{\odot}<M_{\mathrm{i}}<1.2 M_{\odot}$, weighed by the initial mass function of Kroupa et al. (1993). In contrast to Paper I, we do not consider an age-metallicity relation to calculate the range of masses given that MACHO 47.2496.8 belongs to the LMC and agemetallicity relations for the LMC are uncertain due to the low precision of age estimates (see e.g. Cole et al. 2005). In addition, if we apply the age-metallicity relation from Cole et al. (2005) the most massive AGB objects obtained with the metallicity of MACHO 47.2496.8 are not massive enough to experience dredge-up (about $0.85 M_{\odot}$ in their zero age main sequence).

The synthetic models are carried out applying the prescription of Vassiliadis \& Wood (1993) to account for the mass loss. We employ the free-parameter values found in Paper I, which provide the best match for the overall properties of Galactic $s$-enhanced AGB and post-AGB stars: a shift of the minimum core mass for TDU $\Delta M_{\mathrm{c}}^{\min }=-0.065 M_{\odot}$ with respect to the models of Karakas et al. (2002), a minimum asymptotic TDU efficiency $\lambda_{\min }=0.2$, and a ${ }^{13} \mathrm{C}$-pocket size, given as a fraction of the intershell mass $f^{13} \mathrm{C}$,IS $=1 / 40$. In particular, the relatively high $\lambda_{\min }$ is needed in order to match the number of $s$-process enhanced Galactic post-AGB star that are also carbon rich (see Paper I). Once the TDU parameters are fixed, observed $s$-process enhancements such as the $[\mathrm{Zr} / \mathrm{Fe}]$ ratio in Galactic post-AGB stars, can be matched by adjusting $f^{{ }^{13} \mathrm{C}, \text { IS }}$ to a somewhat smaller value than that usually employed in single star models with less efficient TDU (Gallino et al. 1998; Goriely \& Mowlavi 2000). With these choices of the free parameters the abundances observed in all the other types of intrinsic AGB $s$-enhanced stars (namely MS, S, SC and C stars) are also reproduced (see Paper I).

The parameters described above affect the overall enhancement of $s$-process elements with respect to $\mathrm{Fe}$ at the stellar surface, i.e., $[1 \mathrm{~s} / \mathrm{Fe}]$ and $[\mathrm{hs} / \mathrm{Fe}]$. However, they do not affect the relative distribution of $s$-process abundances, represented by the $[\mathrm{hs} / \mathrm{ls}]$ and $[\mathrm{Pb} / \mathrm{hs}]$ ratios, which is mainly a function of ${ }^{13} \mathrm{C}_{\mathrm{eff}}$. In Paper I we found that, for Galactic objects with $[\mathrm{Fe} / \mathrm{H}] \gtrsim-1$, ${ }^{13} \mathrm{C}_{\text {eff }}$ ranges between approximately $2 / 3$ and $4 / 3$ of the standard value introduced by Gallino et al. (1998, see details in Paper I), while a value reduced by roughly a factor of 6 to 12 is needed to fit $\mathrm{Pb}$ stars, which are lower metallicity, extrinsically enriched halo objects. Here we compare our results with a range of values for this parameter to the observational data of MACHO 47.2496.8.

\section{Results}

We select $s$-process enhanced post-AGB stars from our models by choosing those TP-AGB objects that have $[1 \mathrm{~s} / \mathrm{Fe}] \geq 0.25$ or $[\mathrm{hs} / \mathrm{Fe}] \geq 0.25$ and an envelope mass $\leq 0.03 M_{\odot}$. Figure 2 shows stellar population results computed with different ${ }^{13} \mathrm{C}_{\mathrm{eff}}$ values and compared to the observed $s$-process element ratios of MACHO 47.2496.8 measured by Reyniers et al. (2007). The grey scale measures the distribution of stars over the plotted $s$-process ratio in terms of metallicity. The darker area represents 

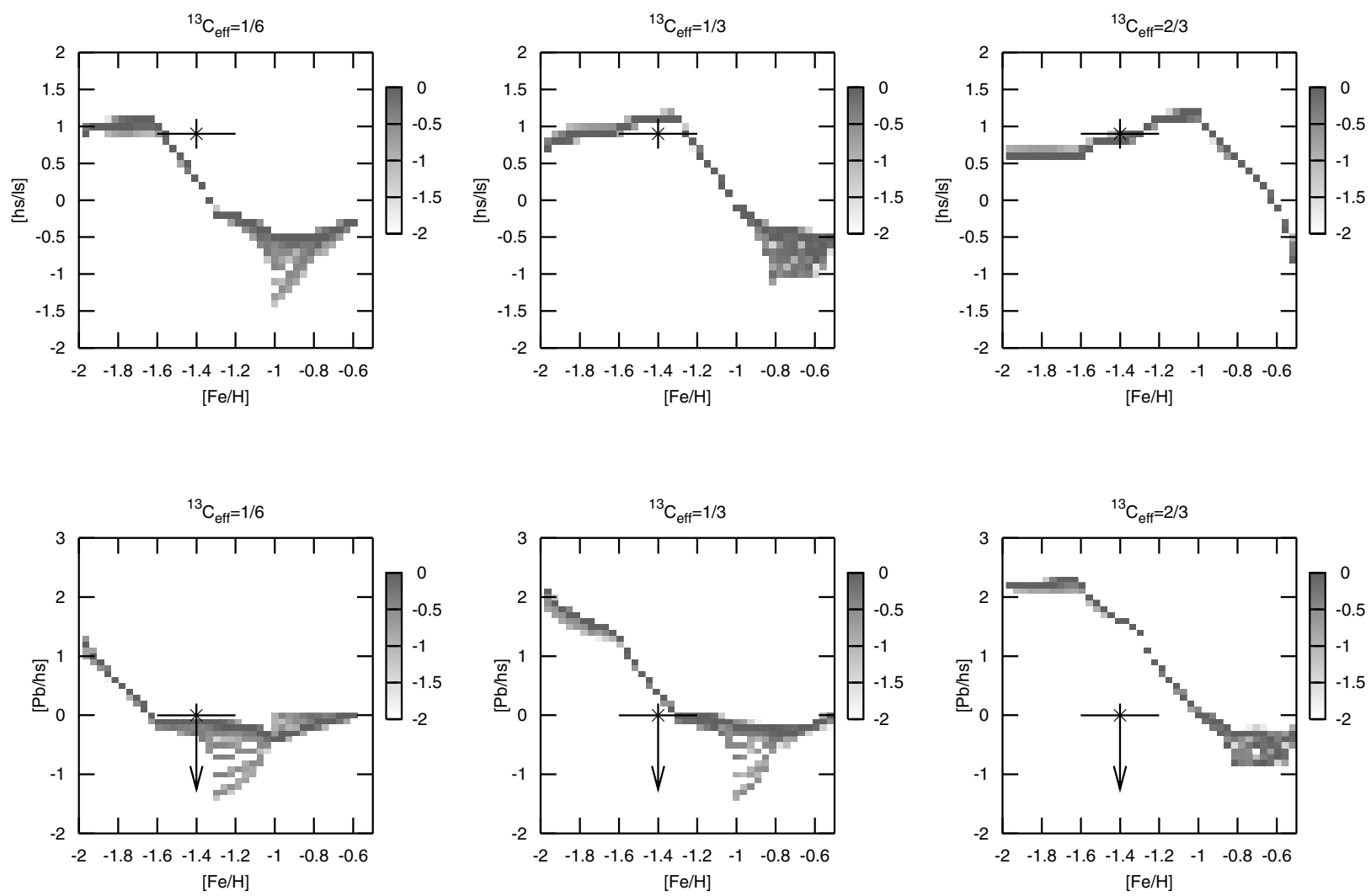

Fig. 2. Distribution of $[\mathrm{hs} / \mathrm{ls}]$ (upper panels) and $[\mathrm{Pb} / \mathrm{hs}]$ (lower panels) ratios in synthesized populations of intrinsically $s$-process enhanced post-AGB stars, calculated with different values of ${ }^{13} \mathrm{C}_{\mathrm{eff}}$, as indicated over each panel. The grey scale is a logarithmic measure of the number distribution of stars over the plotted $s$-process index. The number density is weighed by the initial mass function and by the time each star spends in an abundance bin, and then normalized for each metallicity. The results are compared to the $s$-process element ratios observed in MACHO 47.2496.8, indicated by the crosses. The size of the vertical and horizontal lines indicate the observational errors of the data and the arrow indicates that the observed $[\mathrm{Pb} / \mathrm{hs}]$ ratio is an upper limit.

the contribution of stars with initial mass $M_{\mathrm{i}} \approx M_{\odot}$, which are the most numerous according to the initial mass function that we consider. A good match to the observations for both [hs/ls] and $[\mathrm{Pb} / \mathrm{hs}]$ ratios is obtained with ${ }^{13} \mathrm{C}_{\text {eff }} 1 / 3$ to $1 / 6$ of the standard value. Any smaller ${ }^{13} \mathrm{C}_{\text {eff }}$ value is inconsistent given that the pattern of [hs/ls] as a function of metallicity would shift towards lower metallicities (for a detailed description see Paper I).

The models reported in Fig. 2 can also be employed to interpret the composition of the Galactic post-AGB star IRAS $07134+1005$. This object has metallicity $[\mathrm{Fe} / \mathrm{H}] \sim-1$ and a very high heliocentric velocity (Van Winckel \& Reyniers 2000), suggesting that it belongs to the Galactic halo rather than the disc. In Paper I it was discussed that this object "apparently needs a somewhat smaller ${ }^{13} \mathrm{C}_{\mathrm{eff}}$ " than Galactic post-AGBs of higher metallicity; however, because of the age-metallicity relation employed in Paper I it was not possible to model post-AGB stars of such low metallicity. With the models presented here, we can now confirm that the [hs/ls] ratios approximately equal to zero observed in IRAS $07134+1005$ can be well matched by ${ }^{13} \mathrm{C}_{\text {eff }}=1 / 3$. The same conclusion holds for the intrinsic halo $\mathrm{C}$ star HD 189711 with $[\mathrm{Fe} / \mathrm{H}]=-1.14$ and $[\mathrm{hs} / \mathrm{ls}]=0.7$ shown in Fig. 4 of Paper I. We also note that this value of ${ }^{13} \mathrm{C}_{\text {eff }}$ is not in disagreement with observations of different types of extrinsic $s$-process stars (such as halo $\mathrm{CH}$ giant, halo yellow symbiotic, and $\mathrm{Pb}$ stars) in the same metallicity range (see Fig. 10 of Paper I).

The $[\mathrm{hs} / \mathrm{Fe}]$ and $[\mathrm{ls} / \mathrm{Fe}]$ ratios of MACHO 47.2496.8 are well reproduced by our results for intrinsically $s$-process enhanced
post-AGB stars with the choice of ${ }^{13} \mathrm{C}_{\mathrm{eff}}=1 / 3$ to $1 / 6$ (the choice of $1 / 3$ is represented in Fig. 3 ) and without the need for any modification of the remaining free parameters, which, as described above, were already set according to the properties of Galactic post-AGB stars.

A problem arises when considering the $\mathrm{C} / \mathrm{O}$ and, in particular, the ${ }^{12} \mathrm{C} /{ }^{13} \mathrm{C}$ ratio in MACHO 47.2496.8. With the high TDU assumed in our model we obtain $\mathrm{C} / \mathrm{O}>10$ and ${ }^{12} \mathrm{C} /{ }^{13} \mathrm{C}$ ratios at least an order of magnitude higher than those observed. A better solution is possible by decreasing $\lambda_{\min }$. For example, using $\lambda_{\text {min }}=0.1$ we obtain $\mathrm{C} / \mathrm{O}>4$ and ${ }^{12} \mathrm{C} /{ }^{13} \mathrm{C}>300$. Then, we need to increase $f_{13} \mathrm{C}$,IS to $1 / 20$ to match the $[1 \mathrm{~s} / \mathrm{Fe}]$ and [hs/Fe] ratios. The problem is that, with this choice, we do not match the number of $s$-process enhanced Galactic post-AGB star that are also carbon rich (see Paper I) and it is difficult to find a consistent solution for these two different constraints. A more promising explanation can be found by remembering that model predictions always produce too high ${ }^{12} \mathrm{C} /{ }^{13} \mathrm{C}$ ratios with respect to observations, both for red giant and AGB stars. Extra-mixing processes, also sometimes called "deep mixing" or cool bottom processing, that would enable envelope material to suffer proton captures, thus transforming ${ }^{12} \mathrm{C}$ into ${ }^{13} \mathrm{C}$, have been invoked to explain, e.g., the low ${ }^{12} \mathrm{C} /{ }^{13} \mathrm{C}$ ratios observed in giant stars (Gilroy 1989) and in carbon stars (Abia et al. 2001), as well as in meteoritic silicon carbide grains from AGB stars (e.g. Zinner et al. 2006). We cannot rule out that such processes could also have affected the C composition of MACHO 47.2496.8. Recently, mixing instabilities have been found to occur in first giant branch 

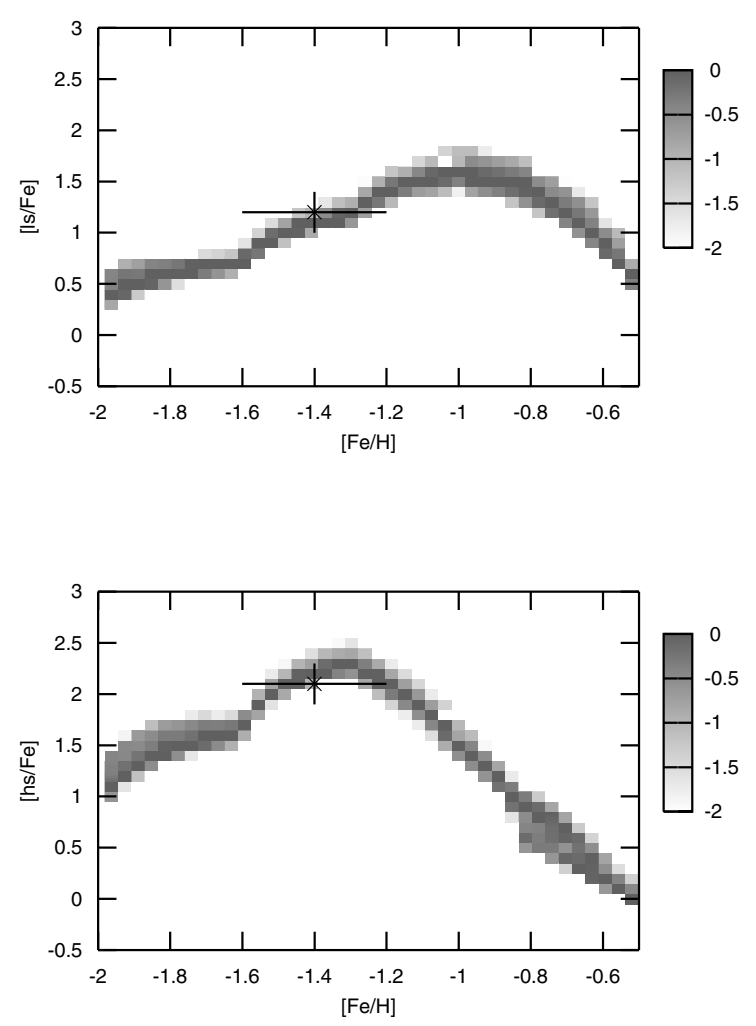

Fig. 3. Distribution of $[1 \mathrm{~s} / \mathrm{Fe}]$ (upper panel) and $[\mathrm{hs} / \mathrm{Fe}]$ (lower panel) ratios in a synthetic population of intrinsically $s$-process enhanced postAGB stars, calculated by assuming ${ }^{13} \mathrm{C}_{\mathrm{eff}}=1 / 3$. The grey scale is a logarithmic measure of the number distribution of stars over the plotted $s$-process index. The number density is weighed as described in the caption of Fig. 2. The results are compared to $[\mathrm{ls} / \mathrm{Fe}]$ and $[\mathrm{hs} / \mathrm{Fe}]$ ratios measured in MACHO 47.2496.8, indicated by the crosses. The size of the vertical and horizontal lines indicate the errors on the data measurements.

stars because of a small inversion in the molecular gradient, just above the H-burning shell, where the ${ }^{3} \mathrm{He}\left({ }^{3} \mathrm{He}, 2 p\right)^{4} \mathrm{He}$ reaction is activated (Eggleton et al. 2006; Charbonnel \& Zahn 2007). This physical mechanism likely leads to modifications of the CNO abundances, as observed, and also would be at work during the AGB phase (M. Cantiello, personal communication). Note that extra mixing on the red giant branch would not be enough to explain the ${ }^{12} \mathrm{C} /{ }^{13} \mathrm{C}$ ratio observed in MACHO 47.2496.8.

\section{Conclusion}

We have compared spectroscopic observations of the post-AGB star MACHO 47.2496.8 in the LMC to results obtained by carrying out stellar population synthesis coupled with $s$-process nucleosynthesis in order to obtain constraints on the physics of AGB stars. The result is that the values of the free parameters needed to match MACHO 47.2496.8 are consistent with the values we found in Paper I for Galactic $s$-process enhanced stars:

1. $\Delta M_{\mathrm{c}}^{\mathrm{min}}=-0.065 M_{\odot}$,
2. $\lambda_{\min }=0.2$, and

3. $f^{13} \mathrm{C}, \mathrm{IS}=1 / 40$.

4. Objects with metallicity $[\mathrm{Fe} / \mathrm{H}] \lessgtr-1$ are generally well reproduced by a lower value of ${ }^{13} \mathrm{C}_{\text {eff }}$ than objects with metallicity $[\mathrm{Fe} / \mathrm{H}] \gtrsim-1$.

The strong agreement among results for different populations and for stars in different galaxies reinforces our confidence in the findings of Paper I. Regarding point (4) above, for IRAS07134+1005 $([\mathrm{Fe} / \mathrm{H}]=-1)$ and HD $189711([\mathrm{Fe} / \mathrm{H}]=$ -1.15 ) we have to lower ${ }^{13} \mathrm{C}_{\text {eff }}$ by roughly a factor of 3 , with respect to higher-metallicity objects, for MACHO 47.2496.8 $([\mathrm{Fe} / \mathrm{H}]=-1.4)$ we have to lower ${ }^{13} \mathrm{C}_{\text {eff }}$ by roughly a factor of 3 to 6 , while for $\mathrm{Pb}$ stars $([\mathrm{Fe} / \mathrm{H}] \lesssim-2)$ we have to lower ${ }^{13} \mathrm{C}_{\text {eff }}$ by roughly a factor of 6 to 12 . This trend requires for an explanation within AGB $s$-process models. We conclude that the heavy element chemical composition of the metal-poor LMC star MACHO 47.2496.8 is consistent with model predictions of an intrinsic $s$-process enhanced star, but that an extra-mixing process is needed to account for the carbon isotopic and $\mathrm{C} / \mathrm{O}$ ratios. Future work will have to address the question of whether data from the still small but expanding set of observed extragalactic AGB stars (see e.g. de Laverny et al. 2006) can be fitted with our current choice of model parameters.

Acknowledgements. M.L. gratefully acknowledges the support of NWO through the VENI grant; M.R. acknowledges financial support from the Fund for Scientific Research - Flanders (Belgium).

\section{References}

Abia, C., Busso, M., Gallino, R., et al. 2001, ApJ, 559, 1117

Bonačić Marinović, A., Izzard, R. G., Lugaro, M., \& Pols, O. 2007, A\&A, in press (Paper I) [arXiv: astro-ph/0703122]

Busso, M., Gallino, R., \& Wasserburg, G. J. 1999, ARA\&A, 37, 239

Charbonnel, C., \& Zahn, J.-P. 2007, A\&A, 467, 15

Cole, A. A., Tolstoy, E., Gallagher, J. S. III, \& Smecker-Hane, T. A. 2005, AJ, 129,1465

de Laverny, P., Abia, C., Domínguez, I., et al. 2006, A\&A, 446, 1107 De Ruyter, S., Van Winckel, H., Maas, T., et al. 2006, A\&A, 448, 641 Edvardsson, B., Andersen, J., Gustafsson, B., et al. 1993, A\&A, 275, 101 Eggleton, P. P., Dearborn, D. S. P., \& Lattanzio, J. C. 2006, Science, 314, 1580 Gallino, R., Arlandini, C., Busso, M., et al. 1998, ApJ, 497, 388

Gilroy, K. K. 1989, ApJ, 347, 835

Goriely, S., \& Mowlavi, N. 2000, A\&A, 362, 599

Herwig, F. 2005, ARA\&A, 43, 435

Karakas, A. I., Lattanzio, J. C., \& Pols, O. R. 2002, PASA, 19, 515

Kroupa, P., Tout, C. A., \& Gilmore, G. 1993, MNRAS, 262, 545

Lugaro, M., Herwig, F., Lattanzio, J. C., Gallino, R., \& Straniero, O. 2003, ApJ, 586,1305

Meixner, M., Gordon, K. D., Indebetouw, R., et al. 2006, AJ, 132, 2268

Pollard, K. R., \& Lloyd Evans, T. 2000, AJ, 120, 3098

Pont, F., \& Eyer, L. 2004, MNRAS, 351, 487

Reyniers, M., \& Van Winckel, H. 2007, A\&A, 463, L1

Reyniers, M., Abia, C., Van Winckel, H., et al. 2007, A\&A, 461, 641

Stephenson, C. B. 1989, Cleveland Publications of the Warner and Swasey

Observatory, Cleveland, Ohio: Case Western Reserve University, 2nd Ed.

Van Winckel, H., \& Reyniers, M. 2000, A\&A, 354, 135

Vassiliadis, E., \& Wood, P. R. 1993, ApJ, 413, 641

Zinner, E., Nittler, L. R., Gallino, R., et al. 2006, ApJ, 650, 350 